\title{
Expected ALICE performance on open heavy flavour and quarkonia
}

\author{
Rachid Guernane* for the ALICE Collaboration \\ Laboratoire de Physique Corpusculaire de Clermont-Ferrand, CNRS-IN2P3 \\ 24. Avenue des Landais, 63177 AUBIÈRE CEDEX FRANCE \\ E-mail: guernane@clermont.in2p3.fr
}

\begin{abstract}
Heavy flavours promise to be valuable probes to elucidate the nature of the hot and dense matter formed in heavy ion collisions. Heavy quarks, produced in initial hard scattering processes, are expected to suffer in-medium energy loss. As for heavy quarkonium states, regeneration processes might counterbalance colour-screening effects. ALICE through a comprehensive heavy flavour program, will address these open questions benefiting from unique LHC conditions: unprecedented large production cross sections and initial temperatures expected to be high enough to dissolve bottomonia. In this talk, we review a selection of significant heavy flavour measurements planned to be performed by ALICE.
\end{abstract}

High- $p_{\mathrm{t}}$ physics at LHC workshop University of Jyväskylä, Finland March, 23-27 2007

${ }^{*}$ Speaker. 


\section{INTRODUCTION}

Heavy flavour production at hadron colliders is, for many years now, a very active field of research providing valuable insights into Quantum ChromoDynamics (QCD) phenomenology $[1,2]$. In nucleon-nucleon (NN) collisions, heavy quark-antiquark pair production should be well described with the parton-model approach since the heavy quark mass can be considered sufficiently large with respect to the fundamental QCD scale [3]. Perturbative QCD cross-sections for heavy flavour production have been computed up to Next-to-Leading-Order (NLO) accuracy [4], even if quantitatively large uncertainties remain, typically a factor $2 \div 3$ at LHC energies [2]. ALICE benchmark cross-sections for heavy flavour production at the LHC computed using a Fixed-Order NLO massive calculation from Mangano et al. [4] are presented in Tab. 1 [2, 5, 6].

Table 1: ALICE benchmark total cross-sections for heavy quark $\left(\sigma_{\mathrm{Q}} \overline{\mathrm{Q}}\right)$ and quarkonium inclusive lepton pair $\left(\sigma_{\mu^{+} \mu^{-}}\right)$production in $\mathrm{Pb}-\mathrm{Pb}$ and p-p collisions. $\sigma_{\mu^{+} \mu^{-}}$are predicted from the Colour Evaporation Model [6, 8]. For $\mathrm{Pb}-\mathrm{Pb}$, parton distribution nuclear modification from the EKS98 parametrisation [7] is included and binary scaling from nucleon-nucleon cross-sections is assumed.

\begin{tabular}{|c|c|c|c|c|c|c|c|c|c|}
\hline Colliding system & \multicolumn{4}{|c|}{$\mathrm{Pb}-\mathrm{Pb}$} & \multicolumn{5}{|c|}{$p-p$} \\
\hline$\sqrt{s_{\mathrm{NN}}}(\mathrm{TeV})$ & \multicolumn{4}{|c|}{5.5} & \multicolumn{5}{|c|}{14} \\
\hline$\overline{\text { Flavour }}$ & $\overline{c c \bar{c}}$ & & 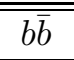 & & & $\overline{c \bar{c}}$ & & $\overline{\overline{b \bar{b}}}$ & \\
\hline Centrality & \multicolumn{4}{|c|}{$\frac{1}{\text { centr. }(5 \%)}$} & \multicolumn{5}{|c|}{ min.-bias } \\
\hline $\begin{array}{l}\sigma_{\mathrm{Q} \overline{\mathrm{Q}}}(\mathrm{b}) \\
N_{\mathrm{Q} \overline{\mathrm{Q}}} \text { per collision }\end{array}$ & $\begin{array}{l}45.0 \\
115\end{array}$ & \multicolumn{3}{|c|}{$\begin{array}{l}1.79 \\
4.56\end{array}$} & & $\begin{array}{l}112 \\
16\end{array}$ & \multicolumn{3}{|c|}{$\begin{array}{c}5.1 \times 10^{-4} \\
0.0072 \\
\end{array}$} \\
\hline State & $\overline{\mathrm{J} / \psi}$ & $\bar{\Gamma}$ & $\overline{\overline{\Upsilon^{\prime}}}$ & $\overline{\overline{\Upsilon^{\prime \prime}}}$ & $\overline{\mathrm{J} / \psi}$ & $\overline{\psi^{\prime}}$ & $\overline{\bar{\Upsilon}}$ & $\overline{\Upsilon^{\prime}}$ & $\overline{\overline{\Upsilon^{\prime \prime}}}$ \\
\hline Centrality & \multicolumn{4}{|c|}{ min.-bias } & \multicolumn{5}{|c|}{ min.-bias } \\
\hline$\sigma_{\mu^{+} \mu^{-}}(\mu \mathrm{b})$ & $\begin{array}{ll}48930 & 879\end{array}$ & 420 & 109 & 61 & 3.18 & 0.0057 & 0.028 & 0.0069 & 0.0041 \\
\hline
\end{tabular}

In heavy-ion collisions, open heavy flavour production is considered to be an interesting probe for studying properties of the dense QCD medium $[9,10]$. Heavy quarks, once produced at the initial stage of the reaction in hard parton interactions, are expected to rescatter and lose energy traversing the surrounding matter, testing this way the expected mass dependence of parton energy loss [11]. In particular, it will be important in ALICE to measure heavy flavoured particle transverse momentum distributions which are expected to be remarkably sensitive to different effects [12]. The low- $p_{\mathrm{t}}(<6 \mathrm{GeV} / c$ at the LHC) region is expected to be sensitive to various non-perturbative effects (such as gluon shadowing, Colour-Glass-Condensate state [14], flow, quark coalescence [13]); while the high- $p_{\mathrm{t}}$ region is expected to be essentially sensitive to jet quenching [15], namely an attenuation (or slowing down) of heavy quark jets after propagation through the dense medium.

In a deconfined medium, quarkonium dissociation due to colour screening has been predicted [16] with dissociation temperatures expected to be ordered according to the hierarchy of quarkonium sizes. Consequently, less tightly bound excited states are predicted to dissolve at lower temperature than their corresponding smaller ground states, leading to a sequential suppression pattern in $\mathbf{J} / \psi$ and $\Upsilon$ yields in nucleus-nucleus collisions as a function of energy density [17]. Recent lattice QCD calculations indicate that hadronic 
resonances could survive even in the deconfined Quark-Gluon Plasma (QGP): $\mathrm{J} / \psi$ and $\eta_{\mathrm{c}}$ could survive up to temperatures $T \simeq 1.6 T_{\mathrm{c}}$ and melt somewhere between $1.6 T_{\mathrm{c}}$ and $1.9 T_{\mathrm{c}}$ [18] while $\eta_{\mathrm{b}}$ state could survive up to $T=2.3 T_{\mathrm{c}}$ [19]. At the LHC, with the increase in available centre-of-mass energy, suitable conditions will be provided for detailed studies to be performed especially in the bottomonium sector: production cross-sections are large (see Tab. 1) both for $\mathrm{p}-\mathrm{p}$ and $\mathrm{Pb}-\mathrm{Pb}$ collisions and initial temperatures are expected to be high enough $\left(T_{0} \simeq 1 \mathrm{GeV}\right.$ for a gluon-dominated plasma formation expected for central $\mathrm{Pb}-\mathrm{Pb}$ at the LHC $[21,22])$ to melt bottomonium states. However, given the large number of uncorrelated $Q \bar{Q}$ pairs initially produced at LHC energies (cf. Tab. 1), this simple step-like suppression pattern could be counterbalanced by the outbreak of coalescence (e.g. recombination mechanisms in statistical [20] and kinetic models [23]) regenerating finalstate quarkonium yields, particularly for charmonium (marginal for bottomonium). Some predictions for the LHC even foresee a quarkonium enhancement instead of a suppression [24].

In what follows, we shall discuss some specific examples of the ALICE potential for the first measurements of open and hidden heavy flavour production at the LHC [6].

\section{ALICE SUBSYSTEMS FOR HEAVY FLAVOUR MEASUREMENTS}

ALICE is a general-purpose experiment equipped with detectors capable of measuring and identifying hadrons, leptons, and photons across a large range of transverse momentum from around $100 \mathrm{MeV} / c$ to about $100 \mathrm{GeV} / c$ [25]. The ALICE design has been optimised to cope with the very demanding environment of high-multiplicity central $\mathrm{Pb}-\mathrm{Pb}$ collisions at LHC energies, where up to 8000 charged particles per rapidity unit at mid-rapidity have been predicted. The studies presented hereafter have been carried out assuming a conservative charged particle rapidity density of $\mathrm{d} N_{\mathrm{ch}} / \mathrm{d} y=6000$ (note that RHIC results tend to favour multiplicity values still lower by about a factor 2.5 [26]). The ALICE detector, presented in Fig. 1, has good acceptance for heavy flavour detection. Heavy flavour decay products are reconstructed in the central barrel $(|\eta|<0.9)$ complemented by the forward muon spectrometer $(-4<\eta<-2.5)$. The ALICE central detectors are embedded in a large magnet (L3) providing a weak solenoidal field $(<0.5 \mathrm{~T})$.

In the ALICE central barrel, charm and beauty particles are selected out of the large background, taking advantage of their long lifetime ${ }^{1}$. Consequently, the ALICE detection strategy relies on resolving secondary detached vertices consisting of identified tracks with large impact parameters $\left(d_{0}\right)$, the impact parameter being the distance of closest approach of a particle trajectory to the primary vertex. Precision on secondary vertex determination is provided by the two innermost layers of the inner tracking system (ITS) made of silicon pixel detectors (SPD). A resolution $\sigma_{d_{0}}(r \varphi)<60 \mu \mathrm{m}$ is achieved for $p_{\mathrm{t}} \gtrsim 1 \mathrm{GeV} / c$. Particle tracking relies on the six concentric layers of high-resolution silicon detectors of the ITS: the two SPD layers quoted above, plus two layers of silicon drift detector (SDD), and two layers of silicon strip detector (SSD), a large volume $\left(\sim 95 \mathrm{~m}^{3}\right)$ time-projection chamber (TPC),

\footnotetext{
${ }^{1} D^{0}$ mesons have proper decay length $c \tau=(123.0 \pm 0.4) \mu \mathrm{m}, D^{ \pm}$mesons have $c \tau=315 \mu \mathrm{m}$, and $B$ mesons of about $500 \mu \mathrm{m}[27]$.
} 


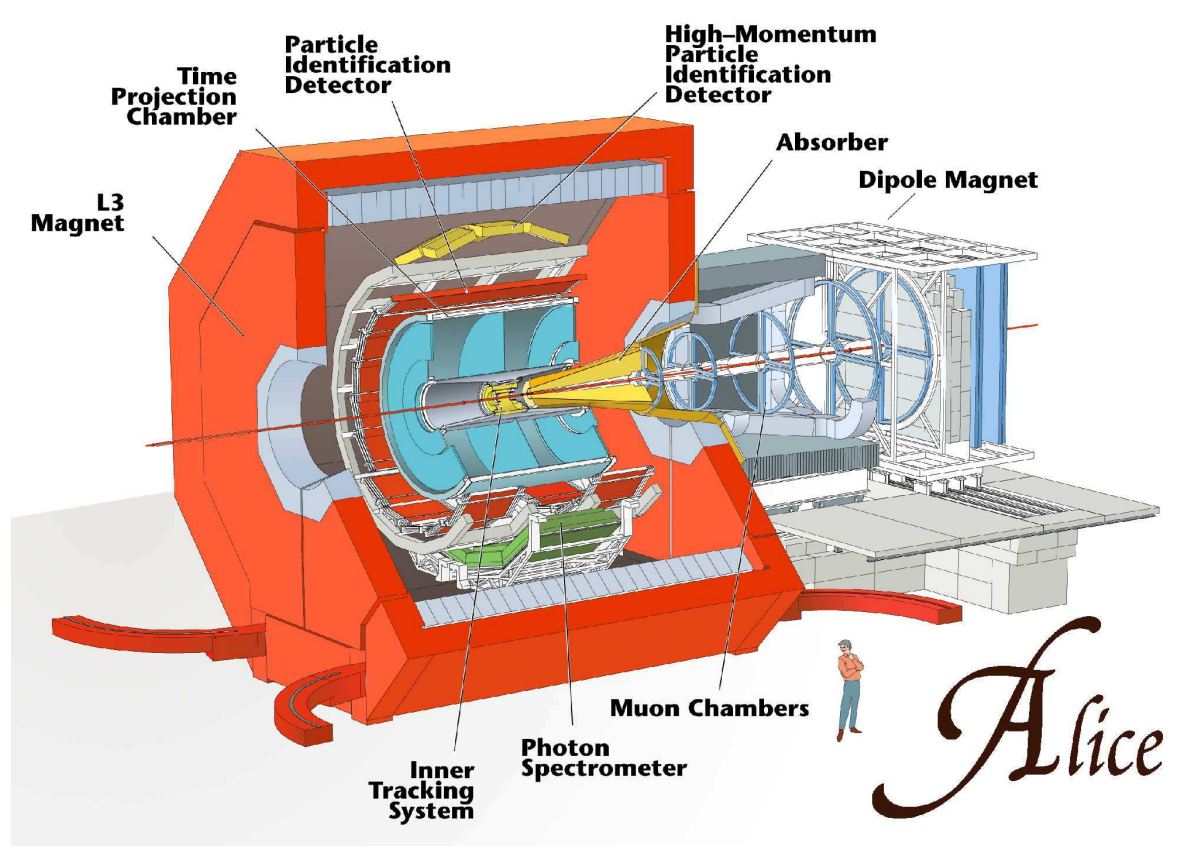

Figure 1: Layout of the ALICE detector.

and a high-granularity transition-radiation detector (TRD) ${ }^{2}$. Particle identification in the central region is performed over the full azimuth by a $\mathrm{d} E / \mathrm{d} x$ measurement in the tracking detectors, a large area high-resolution array of TOF counters, and transition radiation in the TRD. A spectrometer dedicated to muon detection and identification complete the ALICE set-up. The ALICE muon spectrometer consists of a passive front absorber of total thickness corresponding to ten interaction lengths to absorb hadrons and photons from the interaction vertex, a high-granularity tracking system of ten planes of cathode pad chambers, a large dipole magnet creating a field of $0.7 \mathrm{~T}$ (field integral of $3 \mathrm{~T} \cdot \mathrm{m}$ ), and a trigger system made of four planes of resistive plate chambers performing the selection of high transverse momentum muons. Muons penetrating the whole spectrometer length are measured with a momentum resolution of about $1 \div 2 \%$ [28].

\section{CHARM PRODUCTION MEASUREMENT VIA HADRONIC DECAYS}

ALICE capability for measuring direct charm production through the reconstruction of the exclusive hadronic decays have been evaluated using the benchmark $D^{0} \rightarrow K^{+} \pi^{-}$ (and charge conjugate) two-body decay mode of branching ratio $(3.83 \pm 0.09) \%[6,29,30]$. Such a measurement opens the possibility of separating charm from beauty and provides a direct way to access charm transverse momentum spectrum which is of crucial interest when trying to assess the effects induced by the nuclear medium in $\mathrm{p}-\mathrm{Pb}$ and $\mathrm{Pb}-\mathrm{Pb}$ collisions. $\mathrm{A}$ $D^{0}$ candidate consists of a pair of oppositely charged tracks originating from a secondary

\footnotetext{
${ }^{2} \mathrm{TRD}$ is not used as a tracking device in the analysis presented hereafter.
} 


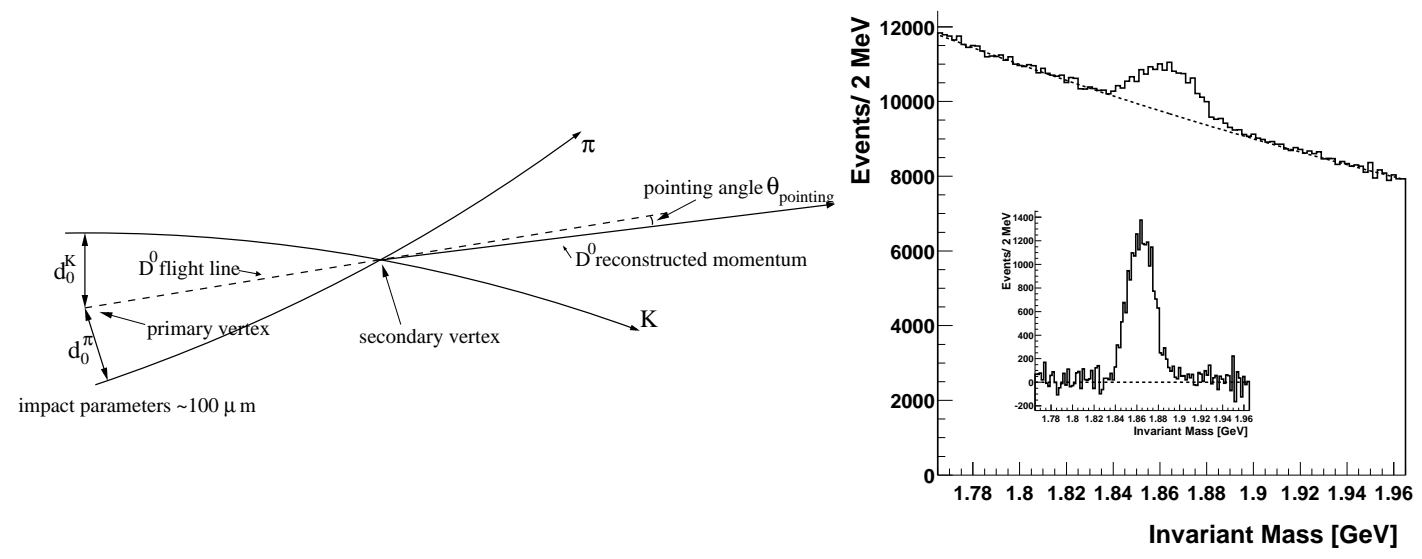

Figure 2: Sketch of the $D^{0} \rightarrow K^{+} \pi^{-}$decay (left hand panel). $K^{+} \pi^{-}$invariant-mass distribution corresponding to $10^{7}$ central $\mathrm{Pb}-\mathrm{Pb}$ events (right hand panel) after selection cuts applied; the background-subtracted distribution is shown in the insert.

vertex (cf. Fig. 2 left hand panel) which has a $K^{+} \pi^{-}$mass in the range $M_{D^{0}} \pm 3 \sigma$ i.e. $|\Delta M|<12 \mathrm{MeV} / c^{2}$ in the present study (cf. Fig. 2 right hand panel).

Extracting a $D^{0}$ signal with a good significance in central $\mathrm{Pb}-\mathrm{Pb}$ collisions requires a drastic selection procedure to reduce the huge combinatorial background by at least $6 \div 7$ orders of magnitude $\left(S / B \sim 10^{-6}\right.$ in the mass range $M_{D^{0}} \pm 3 \sigma$ before any geometrical or kinematical selection). A dedicated set of cuts (distance of closest approach between the tracks, $K$ and $\pi$ minimum transverse momentum, and decay angle) detailed in Ref. [29, 30], improves the $S / B$ by about two orders of magnitude. The $S / B$ ratio is then increased by three additional orders of magnitude imposing a combined cut on the values of $d_{0}^{K} \times d_{0}^{\pi}$ and $\cos \theta_{\text {pointing }}{ }^{3}\left(d_{0}^{K} \times d_{0}^{\pi}<-40,000 \mu \mathrm{m}^{2}\right.$ and $\cos \theta_{\text {pointing }}>0.98$ in this simulation study). Cut tuning has been performed in the present study for each separate $D^{0}$ transverse momentum bin. With $10^{7} \mathrm{~Pb}-\mathrm{Pb}$ events, we expect a $p_{\mathrm{t}}$-integrated significance of 37 and larger than 10 up to $p_{\mathrm{t}} \sim 10 \mathrm{GeV} / c$. The lower $p_{\mathrm{t}}$ limit is expected to be about $1 \mathrm{GeV} / c$ and even lower in p-p collisions (cf. Fig. 3).

\section{BEAUTY MEASUREMENTS FROM SEMILEPTONIC DECAYS}

\subsection{Single inclusive production cross-section measurement using muons}

Beauty production in $\mathrm{Pb}-\mathrm{Pb}$ collisions will be measured in ALICE via semi-muonic and semi-electronic decays in the pseudo-rapidity regions $-4<\eta<-2.5$ and $|\eta|<0.9$ respectively [28]. In the semi-muonic decay channel, both inclusive muons (cf. Fig. 4(c)) and opposite-sign dimuons are considered. The dimuon sample is divided into two topologically distinct contributions: $b$-chain decays (named $B D_{\text {same }}$ in Fig. $4(\mathrm{~d})$ ) of low mass $M_{\mu^{+} \mu^{-}}<$ $5 \mathrm{GeV} / c^{2}$ and high transverse momentum (cf. Fig. 4(a)) and muon pairs where the two muons originate from different quarks $\left(B B_{\text {diff }}\right)$ emitted at large angles resulting in large

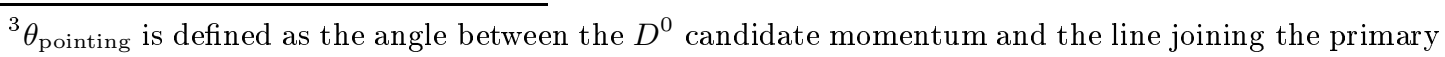
with the secondary vertex as sketched in Fig. 2 on the left hand panel.
} 


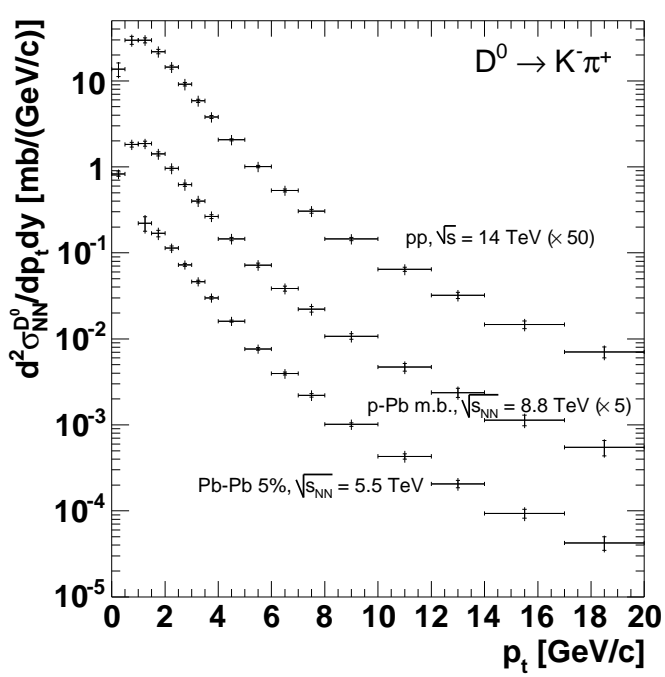

Figure 3: $p_{\mathrm{t}}$-differential cross-section per nucleon-nucleon collision for $D^{0}$ production as expected to be measured with $10^{7}$ central $\mathrm{Pb}-\mathrm{Pb}$ events, $10^{8}$ minimum-bias p- $\mathrm{Pb}$ events, and $10^{9} \mathrm{p}-\mathrm{p}$ minimum-bias events. Statistical (inner bars) and quadratic sum of statistical and $p_{\mathrm{t}}$-dependent systematic errors (outer bars) are shown. An overall normalisation error of $9 \%$ for $\mathrm{Pb}-\mathrm{Pb}, 9 \%$ for $\mathrm{p}-\mathrm{Pb}$ and $5 \%$ for $\mathrm{p}-\mathrm{p}$ is not shown.

invariant masses $M_{\mu^{+} \mu^{-}}>5 \mathrm{GeV} / c^{2}$ (cf. Fig. 4(b)). The beauty signal is enhanced with respect to other sources (charm and $\pi / K$ decay-in-flight) applying a low $p_{\mathrm{t}}$ cut-off set to $1.5 \mathrm{GeV} / c$ in this study. Using Monte Carlo predicted line shapes for $c \bar{c}, b \bar{b}$, and decay background, fits are carried out to find the $b \bar{b}$ fraction in the three different data sets as presented in Fig. 4(a), (b), and (c). Raw muon yields are then converted into inclusive $b$ hadron cross-section following the method initially developed for the UA1 experiment [30]. The expected performance for the measurement of $b$-hadron cross-section for $10^{7}$ central $\mathrm{Pb}-\mathrm{Pb}$ collisions is plotted in Fig. 4(d). b-decay muon statistics is large over the whole $p_{\mathrm{t}}$ range allowing a tight mapping of the production cross-section up to $p_{\mathrm{t}} \sim 20 \mathrm{GeV} / c$.

\subsection{Single inclusive production cross-section measurement using electrons}

Electrons from semileptonic decay of $b$ quarks are characterised by a hard transverse momentum spectrum and a large average impact parameter (typically of a few hundred of microns) with respect to other electron sources: pions misidentified as electrons, decays of primary prompt charmed hadrons, decays of light mesons (e.g. $\pi^{0}$, Dalitz, $\left.\rho, \omega, K\right)$, and photon conversions in the beam pipe or in the ITS inner layers (cf Fig. 5). The ALICE detection strategy will then rely on the selection of displaced tracks identified as electrons.

Electron identification is performed combining information from TRD and TPC. For a TRD electron identification efficiency of $\simeq 90 \%$, a $1 \%$ pion contamination is expected. This efficiency is expected to be roughly constant in the momentum range containing the largest beauty fraction $(1 \div 6 \mathrm{GeV} / c)$. Electrons crossing the TPC are separated from heavier particles by their specific energy loss $\mathrm{d} E / \mathrm{d} x$. Considering only tracks tagged as electrons in the TRD, the contamination from charged kaons and protons is expected to be negligible, 

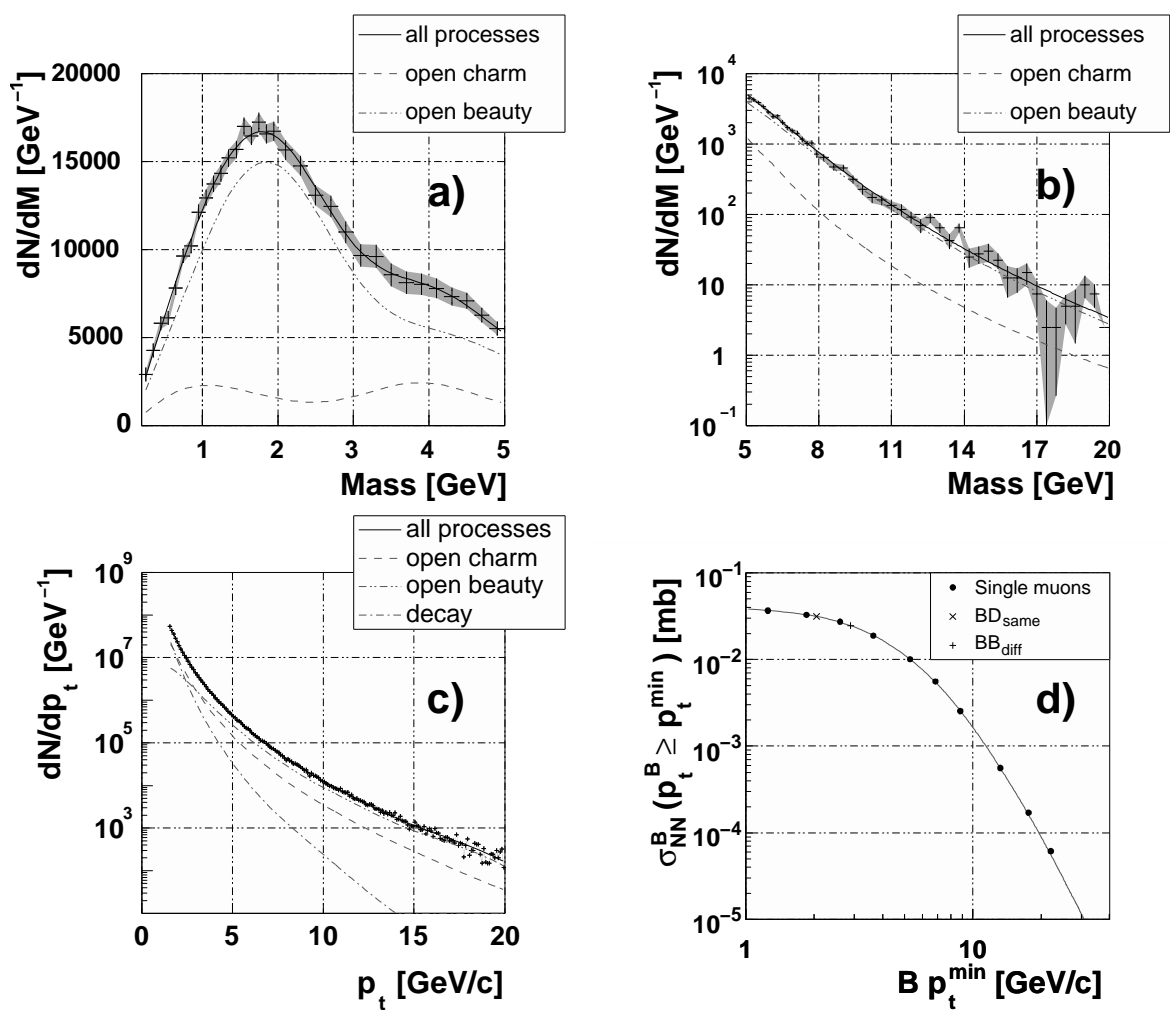

Figure 4: Background subtracted invariant mass distributions of $\mu^{+} \mu^{-}$pairs produced in $10^{7}$ central $\mathrm{Pb}-\mathrm{Pb}$ collisions in the low (a) and high mass regions (b). A $p_{\mathrm{t}}>1.5 \mathrm{GeV} / c$ cut-off has been applied to muon tracks. Charm and beauty signals are plotted in dashed and dotted line respectively. (c) Single muon transverse momentum distribution. (d) Expected performance for the measurement of the inclusive $b$-hadron cross-section in $-4<y_{B}<-2.5$ as a function of $p_{\mathrm{t}}^{\min }$. PYTHIA predictions (solid line) used to produce the signal are also shown.
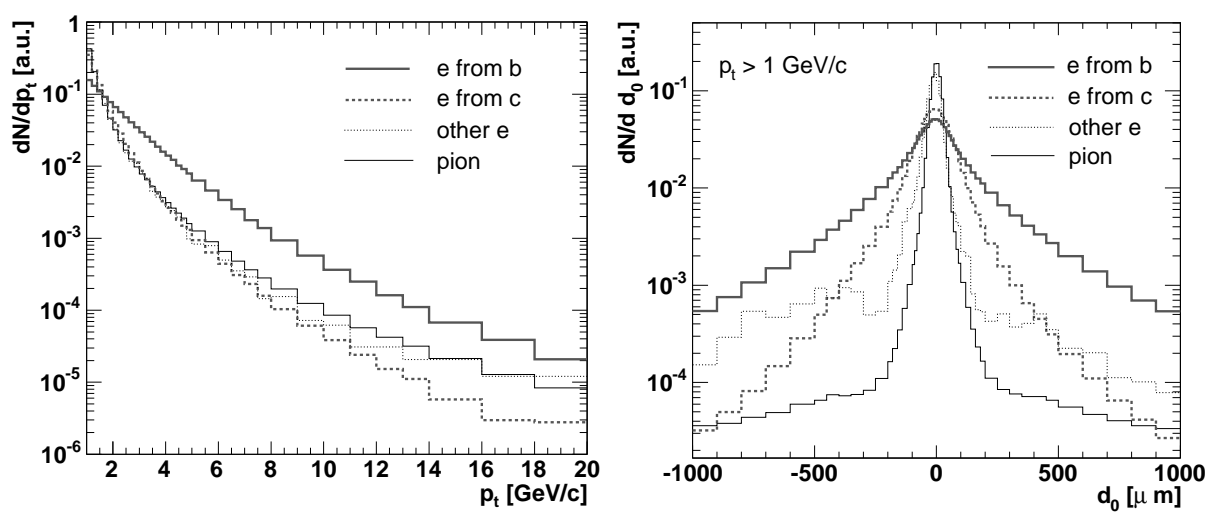

Figure 5: Comparison of electron transverse momentum (left hand panel) and impact parameter projection in the transverse plane (right hand panel) from beauty, charm, light meson, conversion, and pion decays (including a $1 \mathrm{GeV} / c p_{\mathrm{t}}$ cut-off). 

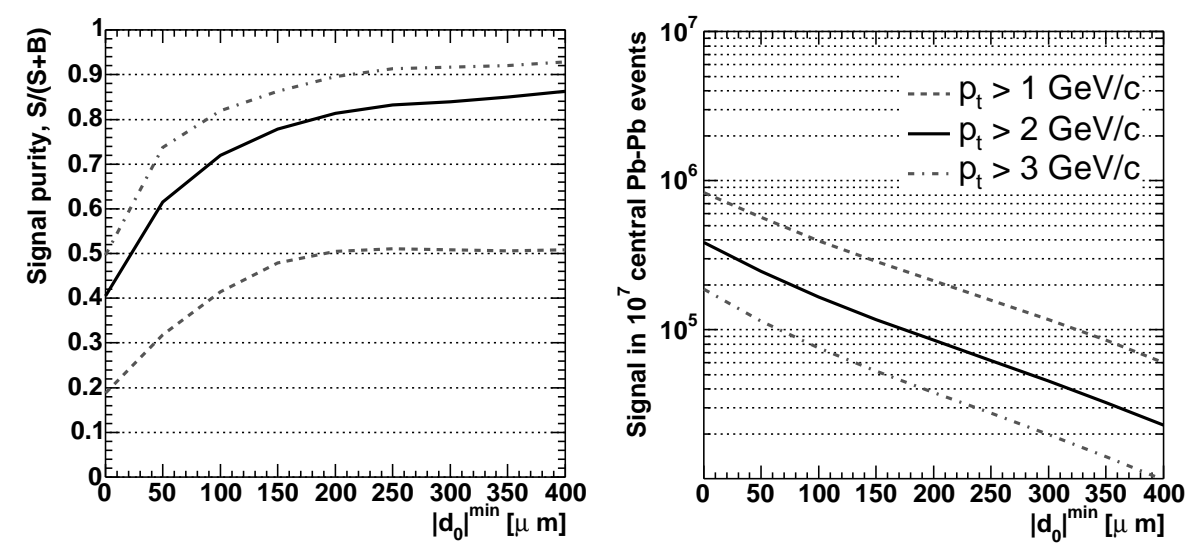

Figure 6: Expected signal purity (left hand panel) and signal (right hand panel) of reconstructed beauty decay electrons as a function of the impact parameter threshold for three different values of transverse momentum cut-off in $10^{7}$ central $\mathrm{Pb}-\mathrm{Pb}$ events.

and electron-tagging probability for electrons is expected to be $\simeq 90 \%$ while electrontagging probability for pions is expected to be at the level of $1 \%$ up to $p \sim 2 \div 3 \mathrm{GeV} / c$ (increasing as a function of $p$ to exceed $60 \%$ for $p \sim 15 \mathrm{GeV} / c$ ). TRD-TPC electron identification is therefore expected to reduces pion contamination by 4 orders of magnitude.

The expected beauty signal purity and statistics as a function of the impact parameter cut-off for different values of the $p_{\mathrm{t}}$ threshold are shown in Fig. 6 left and right hand panel respectively [32]. For instance, $p_{\mathrm{t}}>2 \mathrm{GeV} / c$ and $200<\left|d_{0}\right|<600 \mu \mathrm{m}$ are expected to provide, for $10^{7}$ central $\mathrm{Pb}-\mathrm{Pb}$ collisions, an electron sample of $8 \times 10^{4}$ with a $90 \%$ purity. An upper limit on $d_{0}$ is applied here in order to reduce long lived strange particles and tracks suffering from large angle scatterings in detector materials.

Finally, the $b$-decay electron transverse momentum distribution is obtained after subtraction of the remaining background (cf. Fig. 7 left hand panel). We intend to subtract the remaining background from charm using the hadronic charm production cross-section measurement (described earlier) while the steeply falling $\left(<2 \%\right.$ for $\left.p_{\mathrm{t}} \gtrsim 4 \mathrm{GeV} / c\right)$ decay background (from pions tagged as electrons and other sources) will be estimated with Monte Carlo simulations from the measured charged pion pt distributions. The corresponding $b$ hadron cross-section per unit of rapidity as a function of the minimum transverse momentum $\left(p_{\mathrm{t}}^{\min }\right)$ is inferred "à la" UA1 just like for the muon channel as described in the previous section and the expected performance shown in Fig. 7 (right hand panel). b-quark quenching effects on the $b$-hadron cross-section are also represented in Fig. 7 (right hand panel) but only with a view of illustration since any suppression pattern will be assessed from a comparison with the reference cross-section provided by p-p data. Good performance for semi-electronic beauty detection are also expected in p-p collisions but not covered in these proceedings. For details on p-p studies see Ref. [33].

\section{QUARKONIUM PRODUCTION MEASUREMENTS}

Quarkonia will be detected in ALICE by their decays into both dielectrons and dimuons, 

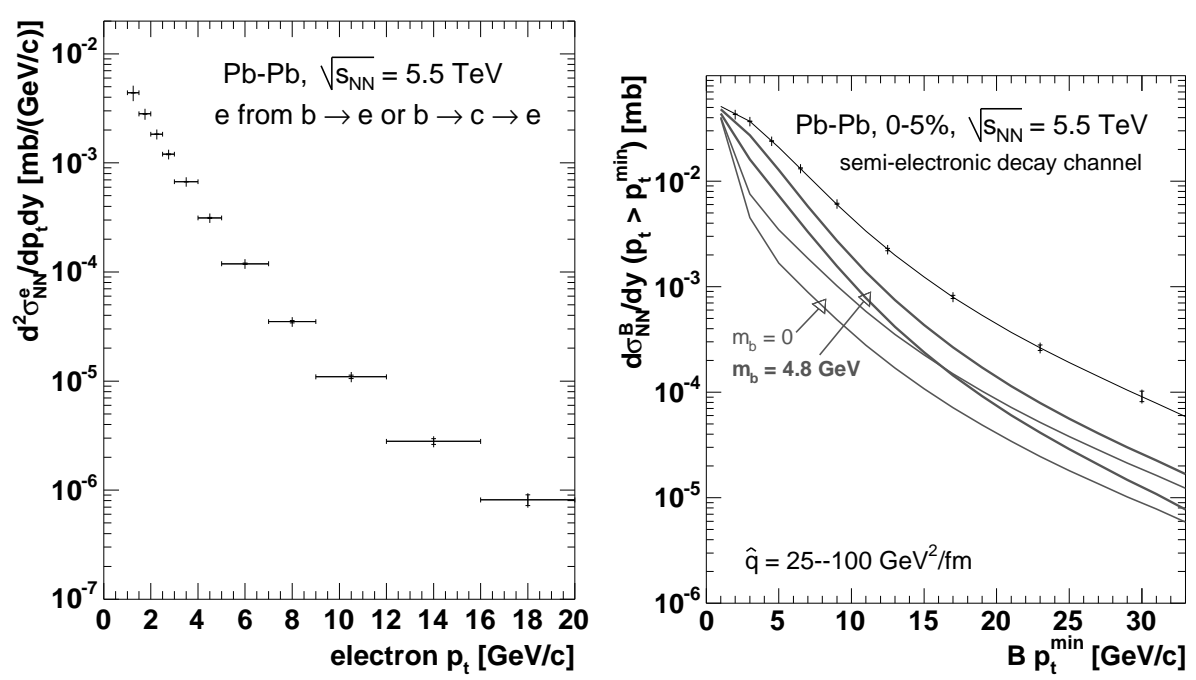

Figure 7: Expected performance for the measurement of the $p_{\mathrm{t}}$-differential $b$-decay electron crosssection per nucleon-nucleon collision as a function of transverse momentum for $10^{7}$ central $\mathrm{Pb}$ $\mathrm{Pb}$ collisions (left hand panel). Statistical (inner bars) and systematic (outer bars) errors are displayed. Corresponding inclusive $b$-hadron cross-section as a function of $p_{\mathrm{t}}^{\min }$ (right hand panel). An overall normalisation error $(9 \%)$ is not included. Quenching predictions from calculation [9] are superimposed for a massive and massless $b$ quark and a transport coefficient $\hat{q}$ in the range $25 \div 100 \mathrm{GeV}^{2} / c$.

in the central barrel and forward muon spectrometer respectively. Description of key detectors involved in quarkonium detection and their expected performance are summarised in Tab. 2. Combined measurements at central and forward rapidities will allow to probe a continuous range of Bjorken- $x$ with values as low as $10^{-5}$, shedding more light on the scale dependence of parton densities in the "small"- $x$ regime.

Expected invariant mass resolution (quoted in Tab. 2) allow to resolve the complete quarkonium spectrum $\left(\mathrm{J} / \psi, \psi^{\prime}, \Upsilon, \Upsilon^{\prime}, \Upsilon^{\prime \prime}\right)$. Resonance signals are extracted integrating invariant mass spectra around the resonance mass, mass windows are fixed to $\pm 2 \sigma$ and $\pm 1.5 \sigma$ for dimuon and dielectron decay channels respectively in these simulation studies (cf. Fig 8 and Fig 9).

Expected quarkonium signal, background, signal-to-background, and signal significance with $500 \mu \mathrm{b}^{-1}$ of integrated luminosity (one year data taking) and for central collisions are presented in Tab. 3 and Tab. 4 in dimuon and dielectron ${ }^{4}$ channels respectively. For charmonium states, despite large expected signal samples $\left(\sim 130,000 \mathrm{~J} / \psi \rightarrow \mu^{+} \mu^{-}\right.$and $\sim 120,000 \mathrm{~J} / \psi \rightarrow e^{+} e^{-}$), expected $S / B$ ratios are relatively small but the expected signal significance is high thanks to the large statistics available. For the $\Upsilon$, the expected signal is roughly two order of magnitude lower than the $\mathbf{J} / \psi$. At the same time, in this high-mass region, the background is lower by around three orders of magnitude, resulting in a signal significance of $\sim 30$ for a one year data taking period. For excited $\Upsilon, \Upsilon(2 S, 3 S)$, even if signal statistics are tight, good performance is still expected. The expected available

\footnotetext{
${ }^{4} \mathrm{~A} 200 \mathrm{~Hz}$ central barrel readout rate is assumed, imposed by the TPC rate capability.
} 
Table 2: Synopsis of quarkonium detection in ALICE. Acceptance coverage: rapidity $(y)$ range and integrated absolute (normalised to the full phase space) value $(\mathcal{A})$, Bjorken- $x$ reach, expected global efficiencies $(\varepsilon)$, and dilepton invariant mass resolution $\left(\sigma_{M_{\ell+\ell^{-}}}\right)$are quoted for p-p and $\mathrm{Pb}-\mathrm{Pb}$ collisions.

\begin{tabular}{|c|c|c|c|c|c|c|c|c|}
\hline \multirow{3}{*}{ ollis } & \multirow{3}{*}{ State } & \multirow{3}{*}{$y$ range } & \multirow{3}{*}{$x$ range } & \multirow{3}{*}{$\mathcal{A}$} & \multirow{2}{*}{\multicolumn{2}{|c|}{$\varepsilon$}} & \multirow{2}{*}{\multicolumn{2}{|c|}{$\sigma_{M_{\ell^{+} \ell^{-}}}\left(\mathrm{MeV} / \mathrm{c}^{2}\right)$}} \\
\hline & & & & & & & & \\
\hline & & & & & $p-p$ & Centr. $\mathrm{Pb}-\mathrm{Pb}$ & $\mathrm{p}-\mathrm{p}$ & Centr. $\mathrm{Pb}-\mathrm{Pb}$ \\
\hline \multirow[t]{2}{*}{$\begin{array}{l}\text { Muon spectrometer } \\
\qquad \begin{array}{l}\quad \text { decay channel } \mu^{+} \mu^{-} \\
\quad \triangleright B=0.7 \mathrm{~T} \\
\quad \triangleright p_{\mu}>4 \mathrm{GeV} / c\end{array}\end{array}$} & $\mathrm{~J} / \psi$ & \multirow{2}{*}{$-4<y<-2.5$} & $\begin{array}{c}2.7 \times 10^{-3}<x_{1}^{\mathrm{p}-\mathrm{p}}<1.2 \times 10^{-2} \\
4.1 \times 10^{-6}<x_{2}^{\mathrm{p}-\mathrm{p}}<1.8 \times 10^{-5} \\
7.0 \times 10^{-3}<x_{1}^{\mathrm{Pb}-\mathrm{Pb}}<3.1 \times 10^{-2} \\
1.0 \times 10^{-5}<x_{2}^{\mathrm{Pb}-\mathrm{Pb}}<4.6 \times 10^{-5}\end{array}$ & 0.0446 & 0.67 & $\underbrace{+1}$ & 72 & 70 \\
\hline & $\Upsilon$ & & $\begin{array}{c}8.2 \times 10^{-3}<x_{1}^{\mathrm{p}-\mathrm{p}}<3.7 \times 10^{-2} \\
1.2 \times 10^{-5}<x_{2}^{\mathrm{p}-\mathrm{p}}<5.5 \times 10^{-5} \\
2.1 \times 10^{-2}<x_{1}^{\mathrm{Pb}-\mathrm{Pb}}<9.3 \times 10^{-2} \\
3.1 \times 10^{-5}<x_{2}^{\mathrm{Pb}-\mathrm{Pb}}<1.4 \times 10^{-4}\end{array}$ & 0.0441 & 0.89 & $\bigcirc^{\circ}$ & 99 & 115 \\
\hline $\begin{array}{l}\text { Central barrel } \\
\qquad \begin{array}{l}\triangleright \text { decay channel } e^{+} e^{-} \\
\triangleright B=0.5 \mathrm{~T} \\
\triangleright \quad e \text { identification with } \\
\quad \operatorname{TRD}(p>1 \mathrm{GeV} / c)\end{array}\end{array}$ & $\mathrm{J} / \psi$ & \multirow{2}{*}{$|y|<0.9$} & $2.3 \times 10^{-4}<x_{1,2}^{\mathrm{Pb}-\mathrm{Pb}}<1.4 \times 10^{-3}$ & 0.295 & & & & 90 \\
\hline $\begin{array}{r}\triangleright e \text { identification with } \\
\operatorname{TRD}(p>1 \mathrm{GeV} / c) \\
\triangleright p \text { measurement with } \\
\text { ITS }+ \text { TPC + TRD }\end{array}$ & $\Upsilon$ & & $7.0 \times 10^{-4}<x_{1,2}^{\mathrm{Pb}-\mathrm{Pb}}<4.2 \times 10^{-3}$ & 0.266 & & & & 33 \\
\hline
\end{tabular}



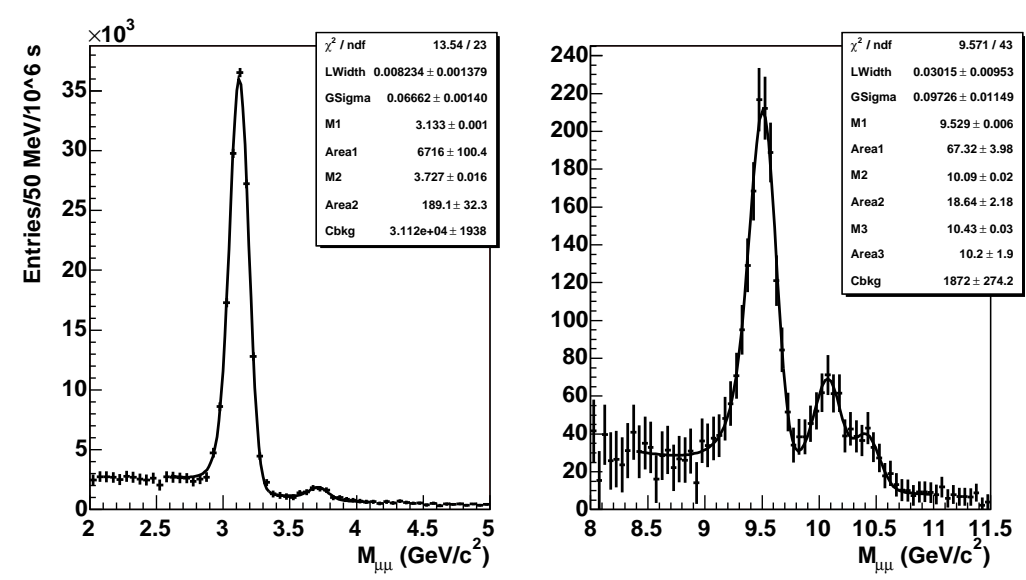

Figure 8: Fits of the opposite-sign dimuon mass yields both in the $\mathbf{J} / \psi$ (left panel) and $\Upsilon$ mass regions corresponding to an integrated $\mathrm{Pb}-\mathrm{Pb}$ luminosity of $500 \mu \mathrm{b}^{-1}$ and for the $4.4 \%$ most central collisions. Quarkonium signal is fitted with Landau convoluted Gaussian functions (solid lines). No suppression or enhancement has been assumed.
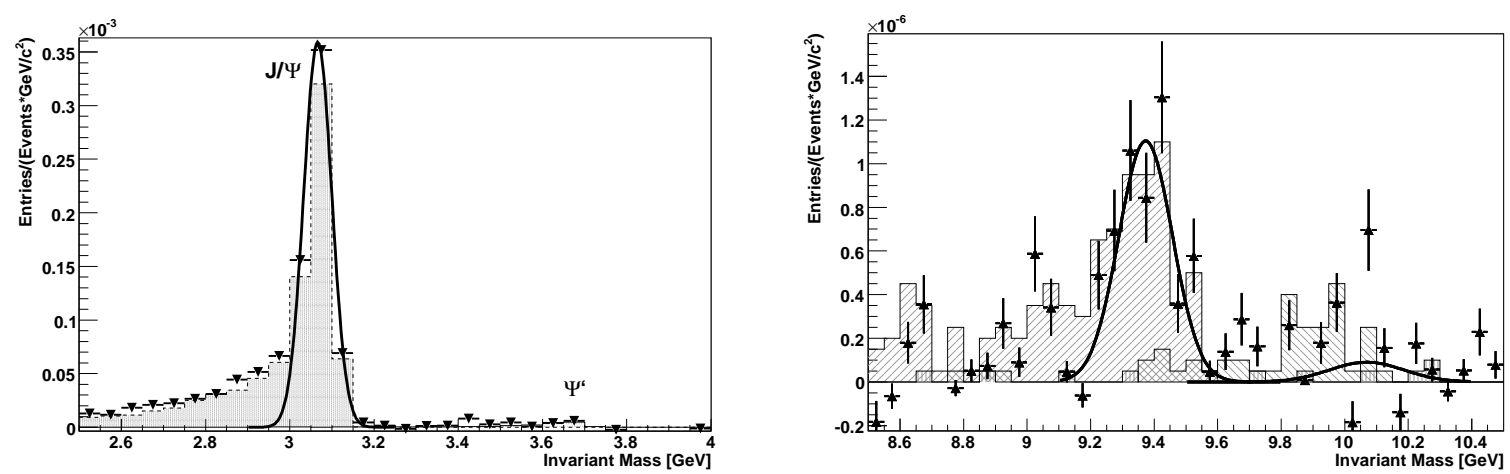

Figure 9: Combinatorial background subtracted opposite-sign dielectron invariant-mass distribution (symbols) in the $\mathbf{J} / \psi$ mass region (left panel) and in the $\Upsilon$ mass region (right panel) for $7.5 \times 10^{7}$ central $\mathrm{Pb}-\mathrm{Pb}$ collisions. Quarkonium signal is fitted with Gaussian functions (solid lines). Filled histograms account for the expected signal yields. No suppression or enhancement has been assumed.

statistics after one year of data taking, should enable us to study centrality dependence of hidden-to-open heavy flavour ratios $(\mathrm{J} / \psi$ and $\Upsilon$ to opposite sign muon pairs from beauty decays) and $p_{\mathrm{t}}$ dependence of quarkonium production needed to constrain QGP models and suppression scenarios [6].

\section{SUMMARY}

With its excellent tracking, vertexing, and particle identification capabilities, ALICE has promising perspectives for open and hidden heavy flavour measurements with abilities to: fully reconstruct hadronic charm decay topologies and address (semi)leptonic heavy quark and quarkonium decays in $\mathrm{p}-\mathrm{p}, \mathrm{p}-\mathrm{Pb}$ and $\mathrm{Pb}-\mathrm{Pb}$ collisions. Channels discussed in 
Table 3: Charmonium and bottomonium states expected signal $(S)$, background ( $B$ ), signal-tobackground $(S / B)$, and signal significance $(S / \sqrt{S+B})$ in the dimuon invariant mass range $\pm 2 \sigma$ around the resonance peak for the $4.4 \%$ most central $\mathrm{Pb}-\mathrm{Pb}$ collision and an integrated luminosity of $500 \mu \mathrm{b}^{-1}[6]$. Charged particle rapidity density is $\mathrm{d} N_{\mathrm{ch}} / \mathrm{d} y=6000$ at mid-rapidity.

\begin{tabular}{lllll}
\hline State & $S\left(\times 10^{3}\right)$ & $B\left(\times 10^{3}\right)$ & $S / B$ & $S / \sqrt{S+B}$ \\
\hline $\mathrm{J} / \psi$ & 130 & 680 & 0.20 & 150 \\
$\psi^{\prime}$ & 3.7 & 300 & 0.01 & 6.7 \\
$\Upsilon$ & 1.3 & 0.8 & 1.7 & 29 \\
$\Upsilon^{\prime}$ & 0.35 & 0.54 & 0.65 & 12 \\
$\Upsilon^{\prime \prime}$ & 0.20 & 0.42 & 0.48 & 8.1 \\
\hline
\end{tabular}

Table 4: Charmonium and bottomonium states expected signal $(S)$, background $(B)$, signal-tobackground $(S / B)$, and signal significance $(S / \sqrt{S+B})$ in the dielectron invariant mass range $\pm 1.5 \sigma$ around the resonance peak for the $10 \%$ most central $\mathrm{Pb}-\mathrm{Pb}$ collision and an integrated luminosity of $500 \mu \mathrm{b}^{-1}[6]$. Charged particle rapidity density is $\mathrm{d} N_{\mathrm{ch}} / \mathrm{d} y=3000$ at mid-rapidity.

\begin{tabular}{lllll}
\hline State & $S\left(\times 10^{3}\right)$ & $B\left(\times 10^{3}\right)$ & $S / B$ & $S / \sqrt{S+B}$ \\
\hline $\mathrm{J} / \psi$ & 121.1 & 88.2 & 1.4 & 265 \\
$\Upsilon$ & 1.3 & 0.8 & 1.6 & 28 \\
$\Upsilon^{\prime}$ & 0.46 & 0.8 & 0.6 & 13 \\
\hline
\end{tabular}

these proceedings will be supplemented by measuring other observables such dilepton correlations, secondary $\mathrm{J} / \psi$ from $b$ decay, $\mathrm{J} / \psi$ polarisation, and $b$-tagged jets. The assessment of the performance of these additional physics channels is currently underway.

\section{Acknowledgments}

Part of this work was supported by the EU Integrated Infrastructure Initiative HadronPhysics Project under contract RII3-CT-2004-506078.

\section{References}

[1] S. Alekhin et al., [arXiv:hep-ph/0601012], [arXiv:hep-ph/0601013].

[2] M. Bedjidian et al., CERN Yellow Report CERN-2004-009, [arXiv:hep-ph/0311048].

[3] P. Nason, S. Dawson, and R.K. Ellis, Nucl. Phys. B 303 (1988) 607.

[4] M.L. Mangano, P. Nason, and G. Ridolfi, Nucl. Phys. B 373 (1992) 295.

[5] N. Carrer and A. Dainese, [arXiv:hep-ph/0311225].

[6] ALICE: Physics Performance Report, Volume II, ALICE Collaboration: F Carminati et al. 2006 J. Phys. G: Nucl. Part. Phys. 32 1295-2040.

[7] K.J. Eskola, V.J. Kolhinen and C.A. Salgado, Eur. Phys. J. C. 9 (1999) 61.

[8] V.D. Barger, W.Y. Keung, and R.J.N. Phillips, Phys. Lett. B 91 (1980) 253. 
[9] M. Djordjevic, M. Gyulassy, and S. Wicks, Phys. Rev. Lett. 94 (2005) 112301, [arXiv:hep-ph/0410372].

[10] N. Armesto, C.A. Salgado and U.A. Wiedemann, Phys. Rev. D 69 (2004) 114003, [arXiv:hep-ph/0312106].

[11] N. Armesto et al., Phys. Rev. D 71 (2005) 054027, [arXiv:hep-ph/0501225].

[12] A. Dainese, Eur. Phys. J. C 33 (2004) 495-503.

[13] Zi-wei Lin and Denes Molnar, Phys. Rev. C 68 (2003) 044901.

[14] Hirotsugu Fujii, Francois Gelis, and Raju Venugopalan, Nucl. Phys. A 780 (2006) 146-174.

[15] Magdalena Djordjevic [arXiv:nucl-th/07053439].

[16] T. Matsui and H. Satz, Phys. Lett. B 178, 416 (1986).

[17] A. Jakovac et al., Phys. Rev. D 75 (2007) 014506.

[18] M. Asakawa and T. Hatsuda, Phys. Rev. Lett. 92 (2004) 012001.

[19] K. Petrov et al., PoS LAT2005 (2006) 153.

[20] P. Braun-Munzinger and J. Stachel, Phys. Lett. B 490 (2000) 196, [arXiv:nucl-th/0007059].

[21] K.J. Eskola, K. Kajantie, and K. Tuominen, Phys. Lett. B 497 (2001) 39.

[22] K.J. Eskola et al., Nucl. Phys. A 696 (2001) 715.

[23] R. L. Thews, M. Schroedter and J. Rafelski, Phys. Rev. C 63 (2001) 054905, [arXiv:hep-ph/0007323].

[24] A. Andronic et al., [arXiv:nucl-th/0611023].

[25] ALICE: Physics Performance Report, Volume I, ALICE Collaboration: F Carminati et al. 2004 J. Phys. G: Nucl. Part. Phys. 30 1517-1763.

[26] K.J. Eskola et al., Nucl. Phys. A 696 (2001) 715-728.

[27] W.-M. Yao et al., J. Phys. G 33, 1 (2006).

[28] R. Guernane et al., ALICE-INT-2005-018.

[29] N. Carrer, A. Dainese, and R. Turrisi, J. Phys. G: Nucl. Part. Phys. 29 (2003) 575-593.

[30] A. Dainese, Ph.D. Thesis, [arXiv:nucl-ex/0311004].

[31] C. Albajar et al., UA1 Collaboration, Phys. Lett. B 213 (1988) 405; C. Albajar et al., UA1 Collaboration, Phys. Lett. B 256 (1991) 121.

[32] F. Antinori et al., ALICE-INT-2005-033.

[33] F. Antinori et al., ALICE-INT-2006-015. 